\title{
Christian Pelras, Goulien, commune bretonne du Cap Sizun. Entre XIXe siècle et IIIe millénaire
}

\section{Martine Cocaud}

\section{(2) OpenEdition \\ 1 Journals}

Édition électronique

URL : https://journals.openedition.org/abpo/1589

DOI : $10.4000 /$ abpo.1589

ISBN : 978-2-7535-1487-4

ISSN : 2108-6443

Éditeur

Presses universitaires de Rennes

Édition imprimée

Date de publication : 20 septembre 2002

Pagination : $176-177$

ISBN : 978-2-86847-768-2

ISSN : 0399-0826

\section{Référence électronique}

Martine Cocaud, «Christian Pelras, Goulien, commune bretonne du Cap Sizun. Entre XIXe siècle et IIle millénaire », Annales de Bretagne et des Pays de l'Ouest [En ligne], 109-3 | 2002, mis en ligne le 20 septembre 2004, consulté le 22 juillet 2022. URL : http://journals.openedition.org/abpo/1589 ; DOI : https://doi.org/10.4000/abpo.1589 
de la corporation. Une étude plus fine des statistiques à moyen terme suggère d'intéressants transferts de sujets entre " périodiques généraux et locaux ". C'est le cas des études de démographie historique qui se cantonnent à présent aux revues des sociétés savantes. D'un autre côté, la stabilité, sur le long terme, de l'histoire religieuse et culturelle ne tient-elle pas, pour une part, à la " relative sur-représentation " des travaux étrangers dans ces domaines (16,5\% de travaux étrangers en histoire religieuse au cours des quatre dernières décennies)?

Comme précédemment, bien entendu, le triple index (index chronologique, index par matières et table des auteurs) qui met près de 300 pages au service de l'utilisateur, orientera celui-ci vers son objet d'étude : thème, personnage, période ou lieu. Un système de renvois permet, en outre, à partir de la référence à un ouvrage collectif, d'en retrouver les diverses contributions, éparpillées par thèmes et par périodes. De même que ce n'est jamais du temps perdu que de flâner au hasard des rayonnages d'une bibliothèque (quand les fichiers électroniques n'y mettent pas le holà!), gageons que le chercheur pourra toujours en parcourant l'ouvrage découvrir, par surcroît, ce qu'il ne s'attendait pas à y trouver. Chacun sait, d'expérience, qu'il n'est pas rare que la consultation d'un titre apparemment sans rapport avec une enquête en cours amène à soulever des questions qui finissent par s'y rapporter.

Bernard MERDRIGNAC

Christian PelRAS, Goulien, commune bretonne du Cap Sizun. Entre XIX siècle et III millénaire, Rennes, PUR, 2001, 486 p.

Le titre surprend : paradoxe d'un regard à la fois restreint géographiquement - Goulien est une petite commune rurale du Finistère sud - et large chronologiquement, qui se rattache au passé et s'évade vers l'avenir. Il suggère pourtant parfaitement l'objectif et la construction de cet ouvrage publié par les Presses Universitaires de Rennes.

La première partie (320 pages) est la reprise - après quelques modifications - de la thèse d'ethnologie soutenue par Christian Pelras en 1965. Le premier objectif de ce travail était d'observer l'adaptation du monde rural à la modernité afin de compléter, en observant un nouveau lieu, les enquêtes menées sur Plozévet. Pour mener cette enquête à bien, Christian Pelras et sa famille ont pris part à la vie de Goulien pendant deux ans. Cette proximité, facilitée par l'apprentissage du breton, a permis à l'auteur des observations fines de la vie rurale. Après une rapide présentation de la commune, il nous les transmet, regroupées en quatre points : les moyens de subsistance, les conditions concrètes de la vie quotidienne, les traditions, les pratiques et les croyances, et enfin la société. Cette monographie est globale puisque le chercheur cerne l'ensemble de la vie quotidienne, cependant les détails fourmillent. La journée de travail d'un paysan, les intérieurs des maisons, l'alimentation, la façon de cuisiner sont décrits avec minutie; ils nous surprennent parfois : pourquoi au Cap Sizun ne conservet-on pas le sang du cochon lors de la tuerie? Ne fait-on pas de boudin? La galette et la crêpe, bien que différentes, sont ici faites l'une et l'autre à base de blé noir, etc. Les pages qui portent sur les réseaux sont riches : sur les liens familiaux et les comportements matrimoniaux bien sûr, mais aussi sur les relations politiques ainsi que sur les rapports d'entraide au travail. Cette analyse, bien 
argumentée et présentée de façon claire à l'aide de schémas, présente l'ensemble de l'organisation spatiale et sociale de la commune. Seule une monographie pouvait mettre en évidence cette complexité, et, tout en ne préjugeant pas de la difficulté à généraliser, ces précisions, résultant d'observations et d'enquêtes menées pendant vingt-quatre mois avec un enthousiasme et une sympathie que le lecteur perçoit bien, nous apprennent beaucoup sur la vie quotidienne au village dans les années soixante et sur les transformations en cours depuis la Première Guerre mondiale. Cette qualité du regard présente l'intérêt majeur de la première partie de l'ouvrage. Par contre, les pages concernant la vie au $\mathrm{xIX}^{\mathrm{e}}$ siècle, qui reposent essentiellement sur la mémoire des anciens, sembleront sans doute trop rapides, historiens ruralistes et sociologues ayant proposé plus récemment d'autres analyses de la modernisation des campagnes. Les quelques pages intitulées " la fin d'un monde " qui concluent ce travail évoquent d'autres ouvrages écrits plus récemment, tels La fin des terroirs d'Eugène Weber ou La fin des paysans d'Henri Mendras.

La seconde partie du livre concerne la vie à Goulien dans la seconde moitié du xxe siècle. Écrite après deux séjours d'un mois à Goulien, elle est beaucoup plus courte (150 pages). Elle s'organise autour de sept points (la population, l'agriculture, les conditions de vie, la culture, la pratique religieuse, la société, les rapports de Goulien avec l'extérieur). L'auteur cherche à décrire les évolutions récentes : certains thèmes de la première partie sont donc repris, mais de façon beaucoup plus rapide, car les observations et les enquêtes réalisées en l'an 2000 n'ont pas la même minutie que celles du premier séjour. Christian Pelras conclut sur l'érosion rapide de " la civilisation de terroir ", évolution qu'il avait déjà perçue en 1960 mais sans en mesurer toutes les conséquences : malgré la recherche de différenciation identitaire (parler le breton, apprendre les danses), la sociabilité communale, très riche jusque dans les années soixante, ne retrouve son dynamisme passé que lors des périodes électorales. Le propos, venant d'un excellent observateur de la Bretagne est intéressant; on peut toutefois regretter qu'il soit un peu rapide. D'autre part, une bibliographie centrée sur le thème de la modernisation rurale lors des quarante dernières années aurait été la bienvenue.

Martine COCAUD 